\title{
PEDOT:PSS-Containing
}

\section{Nanohydroxyapatite/Chitosan Conductive Bionanocomposite Scaffold: Fabrication and Evaluation}

\author{
Alireza Lari, ${ }^{1}$ Tao Sun, ${ }^{2}$ and Naznin Sultana ${ }^{1,3}$ \\ ${ }^{1}$ Faculty of Bioscience and Medical Engineering, Universiti Teknologi Malaysia, 81300 Skudai, Johor, Malaysia \\ ${ }^{2}$ Miniaturized Medical Devices Program, Institute of Microelectronics, Agency for Science, \\ Technology and Research (A* STAR), Singapore 117685 \\ ${ }^{3}$ Advanced Membrane Technology Research Center (AMTEC), Universiti Teknologi Malaysia, 81300 Skudai, Johor, Malaysia \\ Correspondence should be addressed to Naznin Sultana; naznin@biomedical.utm.my
}

Received 11 May 2016; Revised 31 July 2016; Accepted 2 August 2016

Academic Editor: Ping Xiao

Copyright (C) 2016 Alireza Lari et al. This is an open access article distributed under the Creative Commons Attribution License, which permits unrestricted use, distribution, and reproduction in any medium, provided the original work is properly cited.

\begin{abstract}
Conductive poly(3,4-ethylenedioxythiophene)-poly(4-styrene sulfonate) (PEDOT:PSS) was incorporated into nanohydroxyapatite/chitosan (nHA/CS) composite scaffolds through a freezing and lyophilization technique. The bionanocomposite conductive scaffold was then characterized using several techniques. A scanning electron microscope image showed that the nHA and PEDOT:PSS were dispersed homogeneously in the chitosan matrix, which was also confirmed by energy-dispersive X-ray (EDX) analysis. The conductive properties were measured using a digital multimeter. The weight loss and water-uptake properties of the bionanocomposite scaffolds were studied in vitro. An in vitro cell cytotoxicity test was carried out using mouse fibroblast (L929) cells cultured onto the scaffolds. Using a freezing and lyophilization technique, it was possible to fabricate three-dimensional, highly porous, and interconnected PEDOT:PSS/nHA/CS scaffolds with good handling properties. The porosity was $74 \%$ and the scaffold's conductivity was $9.72 \pm 0.78 \mu \mathrm{S}$. The surface roughness was increased with the incorporation of nHA and PEDOT:PSS into the CS scaffold. The compressive mechanical properties increased significantly with the incorporation of nHA but did not change significantly with the incorporation of PEDOT:PSS. The PEDOT:PSS-containing nHA/CS scaffold exhibited significantly higher cell attachment. The PEDOT:PSS/nHA/CS scaffold could be a potential bionanocomposite conductive scaffold for tissue engineering.
\end{abstract}

\section{Introduction}

In recent years, technology has notably developed and a revolution has occurred in the field of surgical reconstruction and organ replacement using artificial implants to treat the failure or loss of an organ or tissue [1]. The need for synthetic bone with similar biological and chemical properties to natural bone has increased due to the limited availability of natural bone grafts [2]. This limitation is the main motivation for developing artificial composite materials $[3,4]$. Bone tissue engineering has provided a new approach to treating bone ailments. Bone is composed of organic and inorganic phases; therefore, the scaffolds should possess properties such as nontoxicity and biocompatibility; appropriate pore sizes and porosity; appropriate surface properties; biodegradability; suitable mechanical properties $[1,5]$. There are several biocompatible inorganic materials that are commonly used as components of bone, and many natural and synthetic polymers can act as the organic portion of the scaffold's fabrication [6].

In 1957, Fukada and Yasuda discovered that natural bone has electrical properties, namely, piezoelectricity, which can form an endogenous electric field that affects cell proliferation [7]. Recently, scaffold-based tissue engineering has mostly focused on the enhancement of bone healing by electrical stimuli. This effect was induced by using compositions of conductive polymer (CP). Various biocompatible conductive polymers have been used in several medical applications since 1980s [8]. CP has a definite potential to be used in composite scaffolds for bone regeneration $[9,10]$.

Poly(3,4-ethylenedioxythiophene) (PEDOT) is an example of a biocompatible conductive polymer that has been 
used in biomedical applications, particularly in nerve tissue engineering $[8,11,12]$. PEDOT can be doped with poly(4styrenesulfonate) (PSS). This PEDOT:PSS copolymer has a moderate band gap and good stability in the doped state [13]. Studies regarding conductive polymer composite scaffolds, particularly PEDOT:PSS, represent a new approach.

The most commonly studied potential materials in tissue engineering are chitosan (CS) and nanohydroxyapatite (nHA) [14]. The combination of CS and nHA has been shown to possess good biocompatibility and suitable mechanical properties [15]. CS, as a natural polymer, was chosen because of such properties as biocompatibility, biodegradability, nontoxicity, and hydrophilicity $[16,17]$. CS is the product of the deacetylation of chitin and is created from linear polysaccharides composed of glucosamine and $N$-acetylglucosamine units linked by $\beta(1-4)$ glycosidic bonds [16]. The reactive functional groups in chitosan, such as acetamides, hydroxyls, and amines, make it a unique component $[6,18]$. However, limitations in its mechanical properties and bone-bonding bioactivity reduce its applicability in tissue engineering [2]. $\mathrm{nHA}$ is a bioceramic material that has more similarity with natural bone-mineral components. In fact, bioactive compounds like HA bind directly to nearby tissues [19-21].

In an earlier study, the fabrication and characterization of scaffolds based on CS and nHA were reported [14]. The present study reports the fabrication and characterization of bionanocomposite scaffolds using CS, nHA, and conductive PEDOT:PSS polymer. Composite scaffolds based on CS, nHA/CS, and PEDOT:PSS/nHA/CS were fabricated with the desired pore sizes and porosity using a freezing and lyophilization technique, and the properties were evaluated. Moreover, this study focused on the role of PEDOT:PSS in the bionanocomposite scaffold and compared it with CS and nHA/CS scaffolds in terms of cell viability, cell attachment, and cell morphology.

\section{Materials and Methods}

2.1. Materials. Hydroxyapatite nanoparticles were prepared in-house using the nanoemulsion technique [22]. PEDOT:PSS (1.1 wt $\%$ dispersion in water, high-conductive grade) and chitosan (medium molecular weight, 90\% deacetylated) were purchased from Sigma-Aldrich. Phosphatebuffered saline (PBS), glacial acetic acid, sodium hydroxide $(\mathrm{NaOH})$, and all other materials were of analytical grade.

\subsection{Methods}

2.2.1. Fabrication of Scaffolds. To prepare $2.5 \%$ (w/v) CS solution, $0.5 \mathrm{~g} \mathrm{CS}$ was mixed with $20 \mathrm{~mL} 1 \%$ (v/v) acetic acid and stirred continually with a magnetic stirrer until completely dissolved. Then, to fabricate the nHA/CS scaffold, nHA powder $(10 \mathrm{wt} \%)$ was incorporated into $2.5 \%(\mathrm{w} / \mathrm{v})$ chitosan solution, stirred adequately, and homogenized using a handheld homogenizer (IKA Ultra-Turax Model T25, Germany). In order to fabricate the third type of scaffold sample, $2 \%$ (v/v) PEDOT:PSS solution was added into the 10\%-nHAcontaining $2.5 \%(\mathrm{w} / \mathrm{v})$ CS solution, magnetically stirred, and homogenized. Then, the solutions were transferred to glass molds and kept frozen at $-80^{\circ} \mathrm{C}$ for 24 hours. Solidliquid or liquid-liquid phase separation was induced when the mixtures of solutions were kept at $-80^{\circ} \mathrm{C}$. The frozen solutions were lyophilized using a freeze-dryer (LABCONCO Freeze Dry System, USA) for 48 hours. During the lyophilization process, sublimation occurred. During sublimation, the solidified solvent was removed, leaving a porous CS polymer scaffold with nHA and PEDOT:PSS molecules incorporated within the polymer matrix. After lyophilizing, the scaffolds were neutralized with $1 \mathrm{M} \mathrm{NaOH}$ followed by washing with distilled water and then again lyophilized.

\subsubsection{Characterization of Scaffolds}

Microstructures of Scaffolds. The microstructure of the composite scaffolds was observed using a scanning electron microscope (SEM, TM3000) and a field-emission scanning electron microscope (FESEM, SU8020, Hitachi). The samples were sputter-coated with gold prior to examination with SEM or FESEM. The pore sizes of the scaffolds were analyzed from SEM and FESEM micrographs using the Image-J software. At least 20 pores were measured and the average pore size was calculated. The porosity of the scaffolds was measured using a liquid-displacement method based on Archimedes' principle, as described elsewhere [14]. Ethanol was used for the purpose of liquid displacement. The scaffold's initial weight $(w)$ was calculated, and then it was immersed into a fixed volume of liquid $\left(V_{1}\right)$ in a measuring cylinder. A vacuum pump was used to allow the liquid to penetrate into the pores of the scaffolds. The volume of ethanol containing the scaffold was measured $\left(V_{2}\right)$. The scaffold specimen was then removed and the remaining volume of liquid was recorded as $V_{3}$. The density and porosity of the scaffold were calculated using the following equations.

Density of the scaffold:

$$
d=\frac{w}{V_{2}-V_{3}}
$$

Porosity of the scaffold:

$$
\varepsilon=\frac{V_{1}-V_{3}}{V_{2}-V_{3}} .
$$

Energy-Dispersive X-Ray Analysis (EDX). Elemental analysis of the scaffold specimens was performed by an energydispersive X-ray (EDX) analyzer, which was directly connected to the SEM (SEM-EDX). It was used to determine the presence of nHA and PEDOT:PSS in the nanobiocomposite scaffold. In this case, the elements calcium, phosphorus, and sulfur were investigated to verify that the materials had been well combined. Elemental mapping, except for an EDX line scan analysis, was performed on the scaffold composite for further confirmation.

Attenuated Total Reflectance-Fourier Transform Infrared Spectroscopy (ATR-FTIR). Chemical bonding analysis of CS, nHA, and PEDOT:PSS was performed using an ATRFTIR (PerkinElmer 5 Series, USA Model). All spectra were recorded in the range of $4000-650 \mathrm{~cm}^{-1}$. 
Contact Angle Measurement. The wettability of all three types of scaffolds was measured via the contact angle (VCAOptima, AST Inc., USA). For comparison, the measurement was also conducted on solvent-cast nonporous films based on CS, nHA/CS, and PEDOT:PSS/nHA/CS. Deionized (DI) water was used as the test liquid, using a 28-gauge blunt tip needle. $1 \mu \mathrm{L}$ of DI water was dropped on different areas of the surfaces of the scaffolds and thin films, and the contact angle was measured at different time points.

Atomic Force Microscopy (AFM). Using an atomic force microscope (AFM) (SPI-3800N, SII Nanotechnology, Inc.) in dynamic force mode, the surface roughness of the nonporous films was determined. The scanning area was $5 \mu \mathrm{m}$ by $5 \mu \mathrm{m}$ while the scan rate was set at $0.72 \mathrm{~Hz}$. The arithmetic mean value of the surface roughness $(\mathrm{Ra})$ was calculated using the software NanoNavi Station/SPA400 (SII Nanotechnology, Inc.). For each sample, three different locations of the AFM images were taken and the average was calculated.

Conductivity Measurement. The scaffold conductivity was measured using a digital multimeter (VC830L, Victor Instruments, China). In order to determine the conductivity, the scaffold samples (thickness of $2 \mathrm{~mm}$ and diameter of $12 \mathrm{~mm}$ ) were hydrated by DI water and were placed in vacant spaces between two probes to measure the resistance. This procedure was repeated three times for each specimen to obtain the average resistance for each scaffold. The conductivity of the scaffolds was calculated by Pouillet's equation:

$$
\delta=\frac{L}{D \times A} .
$$

$L$ indicates thickness, $D$ indicates diameter, and $A$ is the surface area of the sample, $A=\pi \times(D / 2)^{2}$.

2.2.3. Compressive Mechanical Properties. The compressive mechanical properties of the scaffolds were tested with an Instron mechanical tester (Instron 5848, USA) with a crosshead speed of $0.5 \mathrm{~mm} /$ minute. From each polymer concentration sample, cylindrical specimens (diameter $1.2 \mathrm{~cm}$ and height $0.2 \mathrm{~cm}$ ) were prepared. The compressive modulus was determined from the initial linear region of the stressstrain curve. The yield strength was determined from the cross point of the two tangents on the stress-strain curve around the yield point.

Three specimens from each scaffold were cut from different locations (top, bottom, and middle). Compression testing was performed in the longitudinal direction. The averages and standard deviations were calculated. Two sample independent $t$-tests were performed to determine the statistical significance $(p<0.05)$ of the differences in the mechanical properties.

2.2.4. Swelling and Weight Loss Measurement. The swelling or water-uptake properties were measured for each type of scaffold (diameter $1.2 \mathrm{~cm}$, thickness $0.2 \mathrm{~cm}$ ). The initial weight of each specimen was denoted by $W_{0}$. Then, the samples were immersed in $\mathrm{PBS}(\mathrm{pH}=7.4)$ at $37^{\circ} \mathrm{C}$ using a water bath. After predetermined time intervals, the scaffolds were taken out and excessive water was removed using filter paper. The wet weight of the specimens was denoted by $W_{w}$. The following equation was used for calculating the swelling ratio $(S)$ :

$$
S=\frac{\left(W_{w}-W_{0}\right)}{W_{0}} .
$$

Weight Loss Measurement. In order to measure the weight loss of the scaffolds, the scaffolds were incubated in PBS $(0.01 \mathrm{M})$ at $37^{\circ} \mathrm{C}$ for 30 days. The initial weight of the scaffolds was recorded as $W_{i}$. The scaffolds were removed from PBS at regular time intervals, washed with DI water, frozen, and freeze-dried. The final weight was recorded as $W_{f}$. The weight loss was calculated from the following formula:

$$
\text { Weight loss }(\%)=\frac{\left(W_{i}-W_{f}\right)}{W_{i}} \times 100 \text {. }
$$

\subsubsection{In Vitro Cell Culture Study}

Sterilization of Scaffold. All scaffold specimens in triplicate (diameter $1.2 \mathrm{~cm}$ and width $0.2 \mathrm{~cm}$ ) were kept in a 24-well plate and sterilized. In this process, samples were washed three times using PBS with $1 \%$ penicillin and streptomycin, followed by exposure to UV light for 2 hours.

The cell culture method followed the procedure reported elsewhere [14]. Briefly, a mouse fibroblast cell line (L929, ATCC, USA), according to ISO 10993-5: 2009, was used to measure the cytotoxicity of CS, nHA/CS, PEDOT:PSS/nHA/CS, and tetracycline-loaded PEDOT:PSS/ nHA/CS composite scaffolds. L929 cells were cultured in Eagle's Minimum Essential Medium (EMEM, ATCC ${ }^{\circledR}$ $30-2003^{\mathrm{TM}}$, USA) containing $10 \%(\mathrm{v} / \mathrm{v})$ horse serum and incubated at $37^{\circ} \mathrm{C}$ in an atmosphere of $5 \% \mathrm{CO}_{2}$ and $95 \%$ humidity. At $70-80 \%$ confluence, the cells were detached using $0.25 \%(\mathrm{w} / \mathrm{v})$ trypsin-ethylenediaminetetraacetic acid (trypsin-EDTA) (Invitrogen Co., USA). The cells were then counted by an automated cell counter (Luna ${ }^{\mathrm{TM}}$, Logos Biosystems, USA).

Cell Attachment. The cells were seeded onto each scaffold specimen at a density of $\left(10 \times 10^{3}\right)$ cells/well. The cells were cultured for 24 hours (in triplicate samples). After 24 hours, the scaffolds were transferred to another well, washed with PBS, and then trypsinized, and $500 \mu \mathrm{L}$ EMEM was added. The cell suspension was then centrifuged, and the cells were counted using a hemocytometer (counting chambers, Neubauer-improved, Hirschmann) with the use of an inverted microscope (Eclips TS 100, Nikon).

Live/Dead Assay. Live/dead cell staining (live/dead viability/cytotoxicity kit, Invitrogen Co., USA) was performed in order to determine the viable and nonviable L929 cells adhering to the composite scaffolds at 7 days of culture. Briefly, through the enzymatic conversion of the virtually nonfluorescent cell-permeant calcein-AM to the intensely fluorescent calcein (excitation/emission, $\sim 495 \mathrm{~nm} / \sim 515 \mathrm{~nm}$ ), 


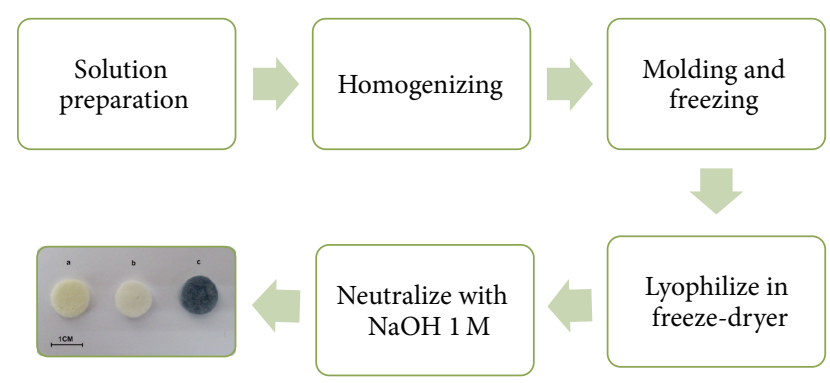

FIGURE 1: Schematic diagram of the fabrication protocol of threedimensional scaffolds with the freezing and lyophilization technique. Notes: $a, b$, and $c$ represent the scaffolds CS, nHA/CS, and PEDOT:PSS/nHA/CS, respectively.

living cells were stained in green. EthD-1 entered the cells and produced a bright red fluorescence in the dead cells (excitation/emission, $\sim 495 \mathrm{~nm} / \sim 635 \mathrm{~nm}$ ) after binding to nucleic acids. After 7 days of culture, L929 cells stained in green and red were observed using a fluorescence microscope (Olympus BX61, Olympus Optical Co., Japan). By counting the number of live cells as well as dead cells and then dividing the number of live cells by the number of total cells, the cell viability was calculated. For the cell viability assessment, at least six areas were randomly selected.

Cell Morphology. L929 cells were cultured at a concentration of $10 \times 10^{3}$ cells/well onto the PEDOT:PSS/nHA/CS scaffold. Then, the 24-well cell culture plate containing the samples was incubated at $37^{\circ} \mathrm{C}$ in an atmosphere of $5 \% \mathrm{CO}_{2}$. At 7 days of cell culture, the cells were washed, fixed, dehydrated, and dried. The morphology of the cells seeded on the composite scaffolds was observed using FESEM.

2.2.6. Statistical Analysis. All data were presented as mean \pm standard deviations (SD). To test the significance, an unpaired Student's $t$-test (two-tail) was applied and a value of $p<0.05$ was considered to be statistically significant.

\section{Results and Discussion}

\subsection{Characterization of Scaffolds}

3.1.1. Morphology. Thermally induced phase separation (TIPS) or freezing and lyophilization is a popular technique to control pore morphology [6]. Usually, it is used for incorporating small molecules into the scaffold. In this technique, firstly, the polymer is dissolved in a solvent to prepare a homogeneous solution, and the solution is cooled in a controlled manner until solid-liquid phase separation is induced. After lyophilization, sublimation of the solvents leaves a porous polymer scaffold. Figure 1 shows a schematic diagram of the fabrication protocol using the freezing and lyophilization technique. In this experiment, three types of scaffolds were fabricated: pure CS, nHA/CS, and PEDOT:PSS/nHA/CS.

Micrographs of the porous scaffolds are shown in Figures 2(a), 2(c), and 2(d). The micrographs display a highly porous scaffold with an interconnected network of spherical pores. The average pore size of the PEDOT:PSS/nHA/CS scaffold was $220 \mu \mathrm{m}$. The pore sizes of the composite scaffolds were found to be in the range of $50 \mu \mathrm{m}$ to $350 \mu \mathrm{m}$. Figure 2(b) displays an FESEM micrograph of the HA nanoparticles. In this investigation, nHA nanoparticles were produced in-house through a nanoemulsion process and subsequent freeze-drying method. nHA contained miniaturized agglomerations of HA nanocrystallites. The size of the nHA was observed to be in the range of $20-30 \mathrm{~nm}$ [14]. It was reported elsewhere that the $\mathrm{Ca} / \mathrm{P}$ ratio was 1.63 as calculated by EDX analysis [14]. The HA nanoparticles were homogeneously distributed in 10\% nHA incorporated into the nHA/CS and PEDOT:PSS/nHA/CS composite scaffolds. It was observed that, with the incorporation of nHA and PEDOT:PSS, the density increased and the porosity decreased (Table 1).

The microstructure of a scaffold is an important factor for cell harboring, penetration, migration, tissue ingrowth, and nutrient supply within the targeted area [23]. The surface properties and surface roughness properties of the scaffolds should be carefully considered, as they can assist cell adhesion and spreading. The chitosan scaffold had more open pores than the other two types. With the incorporation of nHA and PEDOT:PSS, the composite scaffolds possessed irregular and elongated pores together with some closed pores. This occurred during the phase separation process when the composite mixtures were frozen at low temperature and during the subsequent lyophilization process. The porous structure was affected by the crystallization of the solvent phase. The incorporation of nHA and PEDOT:PSS perturbed the phase separation process, and the subsequent lyophilization produced irregularly shaped pores.

3.1.2. EDX Analysis. EDX analyses at different locations of the composite scaffolds confirmed the presence of HA particles inside the pore walls (Figure 2(c)). By EDX elemental analysis, it was found that the ratio of the amount of $\mathrm{Ca}$ to that of $\mathrm{P}$ was 1.60 . The suitable range of the $\mathrm{Ca} / \mathrm{P}$ ratio in $\mathrm{HA}$ is given in the literature as 1.50-1.67 for creating a favorable environment for bone regeneration. An EDX line scan was performed to observe the distribution of sulfur, which was only present in PEDOT:PSS, and in this experiment sulfur was detected in the PEDOT:PSS/nHA/CS composite scaffold. Additionally, elemental mapping was performed to identify the distribution of the HA nanoparticles present in the composite scaffolds. Calcium, phosphorus, and sulfur were dispersed throughout the composite scaffold (Figure 3), which confirms the presence of nHA and PEDOT:PSS.

3.1.3. ATR-FTIR Spectrometry. ATR spectrometry is an appropriate method to evaluate the polymer-particle interaction. The IR spectra shown in Figure 4 contain the typical bands of CS at $1650 \mathrm{~cm}^{-1}$ and $1586 \mathrm{~cm}^{-1}(\theta)$ for the amide I $(\mathrm{C}=\mathrm{O})$ and amide II (NH-) groups, respectively [24]. The peaks at 900 and $1100 \mathrm{~cm}^{-1}$ are related to the saccharine structure. The peaks for aliphatic $\mathrm{C}-\mathrm{H}$ stretching appear at $2996 \mathrm{~cm}^{-1}-882 \mathrm{~cm}^{-1}$, and the $\mathrm{N}-\mathrm{H}$ and $\mathrm{O}-\mathrm{H}$ stretching absorption bands are located in the $3200-3500 \mathrm{~cm}^{-1}$ region [5]. According to the literature describing the IR spectra of 


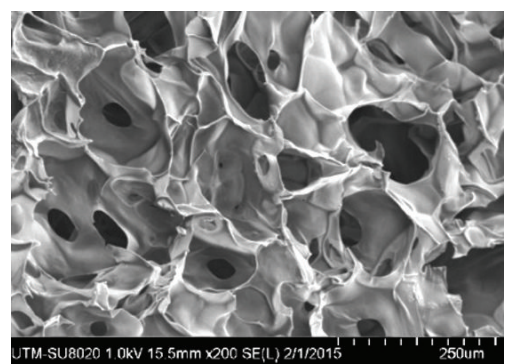

(a)

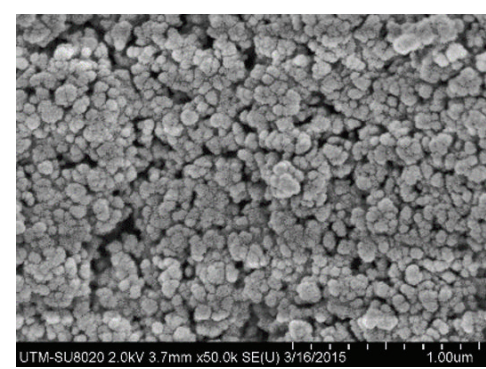

(b)

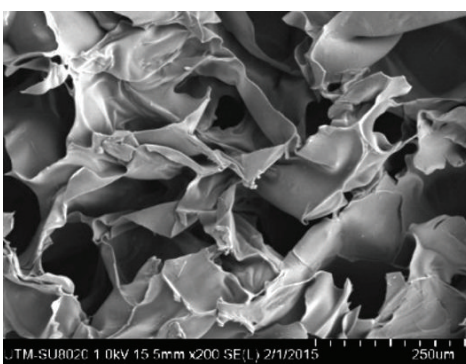

(c)

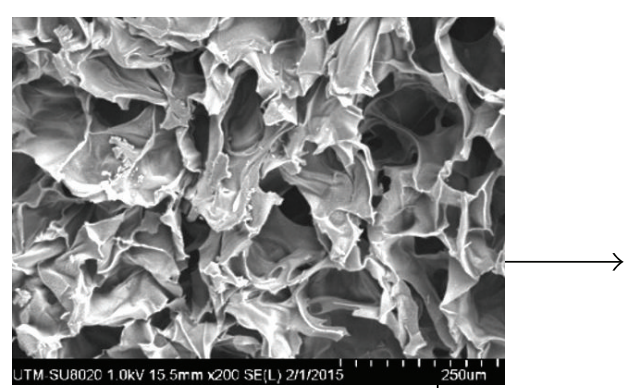

(d)

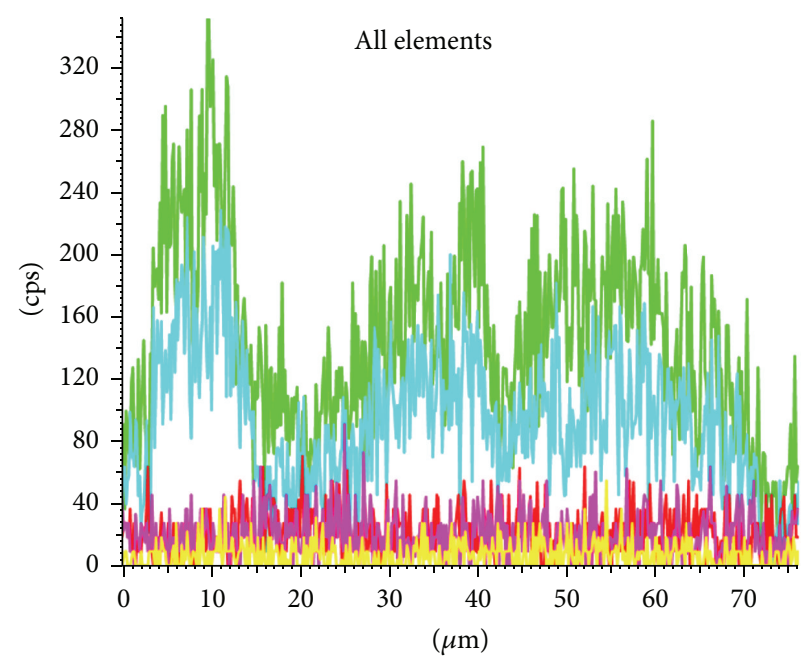

C K $\alpha 1 \_2-\mathrm{P} \mathrm{K} \alpha$

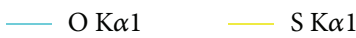

- $\mathrm{Ca} \mathrm{K} \alpha 1$

(e)

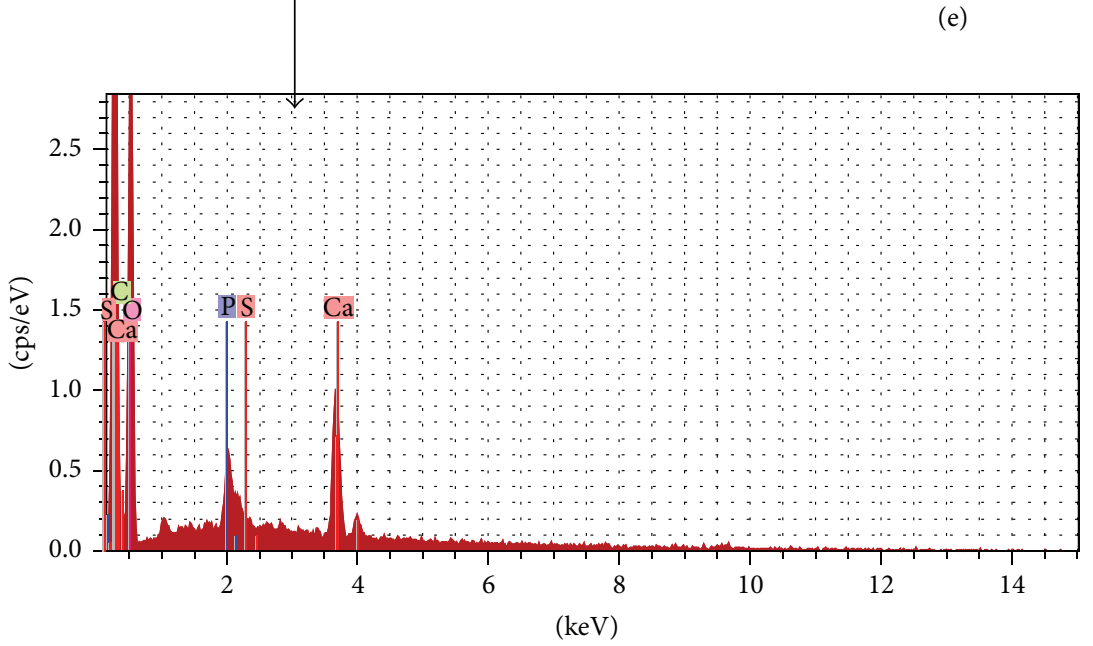

(f)

FIGURE 2: (a) FESEM micrograph of CS scaffold; (b) FESEM micrograph of nHA nanoparticles; (c) FESEM micrograph of nHA/CS scaffold; (d) FESEM micrograph of PEDOT:PSS/nHA/CS scaffold; (e) elemental line scan analysis; and (f) EDX spectrum of PEDOT:PSS/nHA/CS scaffold.

hydroxyapatite, the peaks at $1025 \mathrm{~cm}^{-1}$ and $3750 \mathrm{~cm}^{-1}$ are due to the $\mathrm{OH}$ and phosphate groups of HA [25]. In the IR spectra of the CS/HA composite, there is an intense absorption band at $1050 \mathrm{~cm}^{-1}$, which is related to the phosphate groups. A small band is observed at $3700 \mathrm{~cm}^{-1}$, which is the stretching mode of the hydroxyl group for calcium phosphates, such as HA.

The composite PEDOT:PSS at low concentration showed IR absorption peaks at $1225 \mathrm{~cm}^{-1}$, which are related to the sulfonic acid group of PSS [12]. The basic characteristics of 


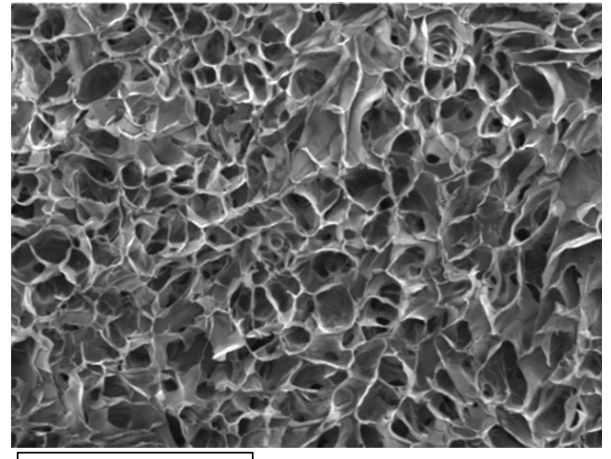

$1 \mathrm{~mm}$

(a)

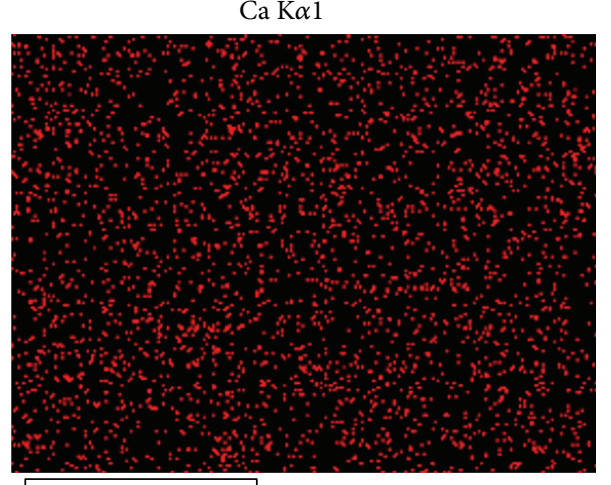

$1 \mathrm{~mm}$

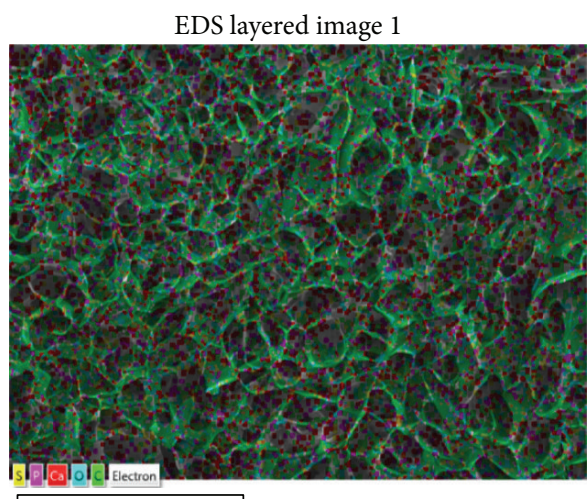

$1 \mathrm{~mm}$

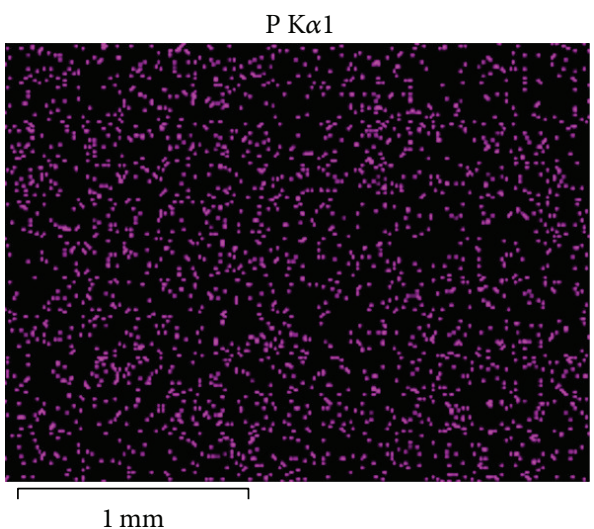

(d)

(c)

$\mathrm{S} \mathrm{K} \alpha 1$

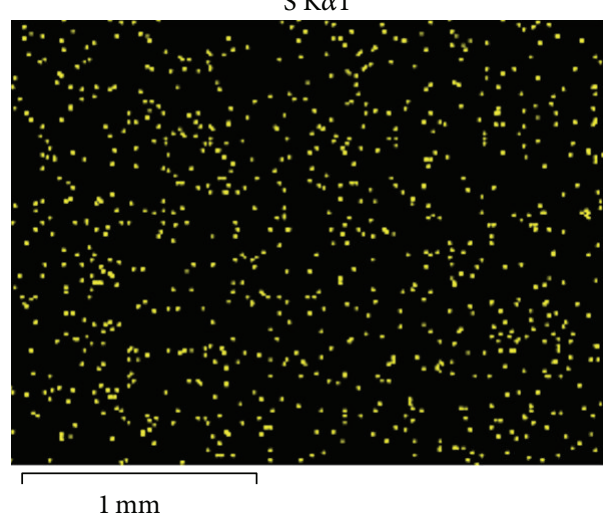

(e)

Figure 3: (a) FESEM and (b-e) elemental mapping of Ca, P, and S in PEDOT:PSS/nHA/CS scaffold.

TABLE 1: Density, porosity, pore sizes, electrical properties, and surface roughness of scaffolds.

\begin{tabular}{lccccc}
\hline Scaffold & Density $\left(\mathrm{g} / \mathrm{cm}^{3}\right)$ & Porosity $(\%)$ & Resistance $(\mathrm{K} \Omega)$ & Conductivity $(\mu \mathrm{S} / \mathrm{m})$ & $\begin{array}{c}\text { Surface } \\
\text { roughness }(\mathrm{nm})\end{array}$ \\
\hline CS & $0.068 \pm 0.005$ & $86 \pm 3.0$ & - & - & $31.28 \pm 4.7$ \\
CS/HA & $0.080 \pm 0.006$ & $77 \pm 3.5$ & - & - & $51.67 \pm 3.5$ \\
CS/HA/PEDOT:PSS & $0.094 \pm 0.008$ & $74 \pm 2.5$ & $9142.0 \pm 715.7$ & $9.72 \pm 0.78$ & $65.96 \pm 3.9$ \\
\hline
\end{tabular}




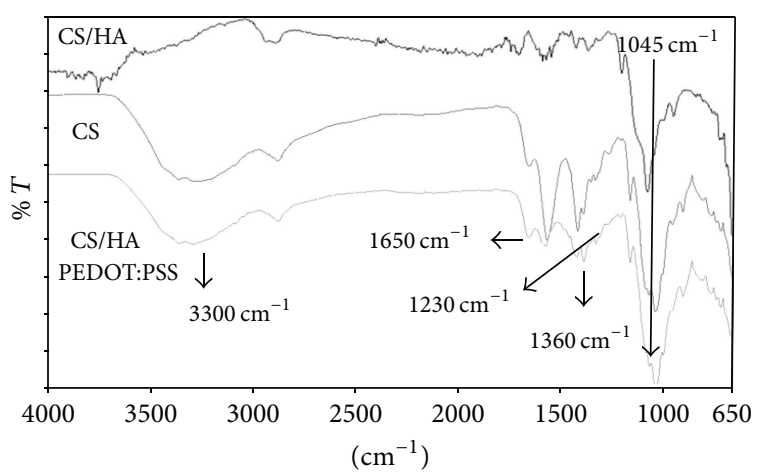

Figure 4: Attenuated Total Reflectance- (ATR-) Fourier Transform Infrared Spectroscopy (FTIR) spectra of CS, nHA/CS, and PEDOT:PSS/nHA/CS scaffolds.

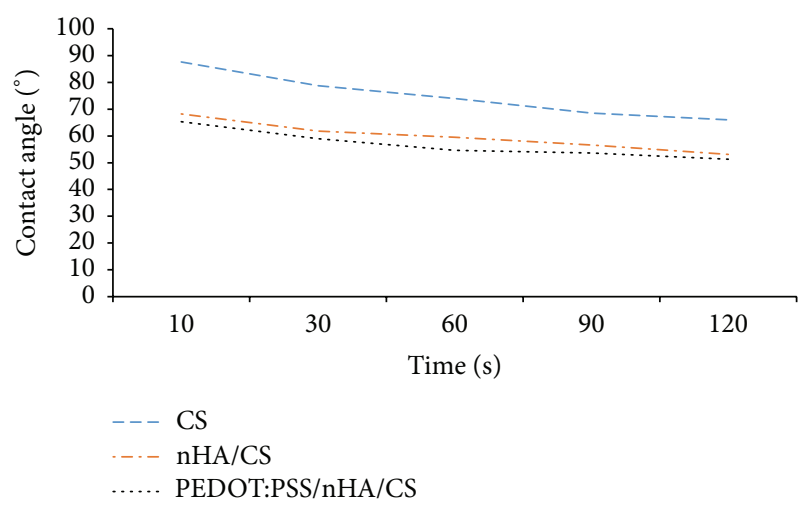

FIGURE 5: The plot of water contact angle values of nonporous thin films versus time.

PEDOT at 935 and $836 \mathrm{~cm}^{-1}$ (C-S stretching), deformation of C-S-C at 690,1090 , and $1230 \mathrm{~cm}^{-1}$ (C-O-C stretching), and $\mathrm{C}-\mathrm{C}$ and $\mathrm{C}=\mathrm{C}$ stretching of the quinoidal structure $\left(1360 \mathrm{~cm}^{-1}\right)$ were observed. All of the PEDOT:PSS peaks in the range of $900 \mathrm{~cm}^{-1}$ to $1200 \mathrm{~cm}^{-1}$ were affected by the absorption of the phosphate groups of HA at $1025 \mathrm{~cm}^{-1}$ [26]. As a result, the ATR data imply that there is a relation between the substrate of the prepared composite and indicate crosslinking of the chitosan chains.

3.1.4. Water Contact Angle. The contact angle measurement is shown in Figure 5 and Table 2. The effect of surface topography on the wettability of the scaffold was reported by other researchers [27]. Porous scaffolds generate space for the penetration of water droplets into the structure and show highly hydrophilic properties. In this study, we used nonporous solvent-cast thin films with the same concentration of CS, nHA, and PEDOT:PSS as used in the scaffolds to evaluate the wettability. Due to the high porosity and large pore sizes, the water droplets were immediately absorbed by the surface of the scaffolds. For the nonporous films, it was found that the contact angle of CS at the start (after $10 \mathrm{~s})$ was $87^{\circ} \pm 10.5^{\circ}$ and then decreased gradually within 2 minutes to $66^{\circ} \pm 7 \cdot 3^{\circ}$. After incorporating hydroxyapatite, the initial wettability of the composite scaffold increased to $68^{\circ} \pm$ $4.1^{\circ}$ at $10 \mathrm{~s}$. The addition of conductive PEDOT:PSS did not change the wettability significantly. The wettability was $65^{\circ} \pm$ $4.6^{\circ}$ for the PEDOT:PSS/nHA/CS scaffold. Due to the amino groups of CS, all the scaffolds were hydrophilic; the composite scaffold containing nHA was more hydrophilic due to the presence of nHA.

3.1.5. Atomic Force Microscopy (AFM). The surface morphology of the composite scaffolds was studied by AFM. The roughness of the composite scaffolds (expressed by the Ra values) is shown in Table 1 . It was observed that the pure CS scaffold had a surface roughness value of $31.28 \pm 4.7 \mathrm{~nm}$. With the addition of HA, the surface roughness increased to $51.67 \pm 3.5 \mathrm{~nm}$. On the other hand, the scaffold containing PEDOT:PSS exhibited a surface roughness of $65.96 \pm 3.9 \mathrm{~nm}$.

It was reported that an increase in surface roughness would enhance the wettability caused by the surface chemistry [28]. In this study, with the addition of nHA and PEDOT:PSS, the surface roughness was increased and the wettability was decreased as observed via the contact angle measurements. According to the literature, particle size and surface topography are able to influence cellular behavior. Deligianni et al. showed that the degree of roughness of the material surface had an effect on cell attachment and that when the surface roughness increased, the number of adherent cells increased [28]. In this study, the results indicated that PEDOT:PSS was able to improve the surface roughness, which is a beneficial factor for cell attachment.

3.1.6. Conductivity Measurement. The conductivity of the prepared scaffold of CS/HA/PEDOT:PSS was measured and calculated by Pouillet's equation. It was observed that the scaffolds containing PEDOT:PSS had conductivities of $9.72 \pm$ $0.78 \mu \mathrm{s} / \mathrm{m}$ (Table 1 ). The other scaffolds without PEDOT:PSS did not exhibit any conductivity. Previous reports described this as being due to the charge-carrier motion inside the PEDOT:PSS grains, which are dispersed in the structure of the scaffold [12]. This electrical conductivity of the bionanocomposite scaffold could enhance the cell-scaffold interaction due to the resulting superior intracellular signaling process [29].

3.2. Compressive Mechanical Properties. Figures 6(a) and 6(b) show the typical compressive stress-strain curves for the different types of scaffolds. The curves exhibited a linear increasing trend, followed by a long plateau and a sudden increase in the densification stage. Similar results were described by other researchers [30,31]. It was found that both the compressive modulus and the yield strength increased with the addition of HA and PEDOT:PSS (Figure 6(c)). With the same processing conditions, the compressive modulus varied from 1.1 MPa to $4.6 \mathrm{MPa}$ and the yield strength varied from $0.2 \mathrm{MPa}$ to $0.48 \mathrm{MPa}$ for the chitosan scaffold and HA/chitosan scaffold, respectively. From the statistical analysis, it was found that both the compressive modulus and the compressive yield strength of the chitosan scaffolds were significantly different from those of the HA/chitosan and HA/chitosan/PEDOT:PSS scaffolds $\left({ }^{*} p<0.05\right.$ and 


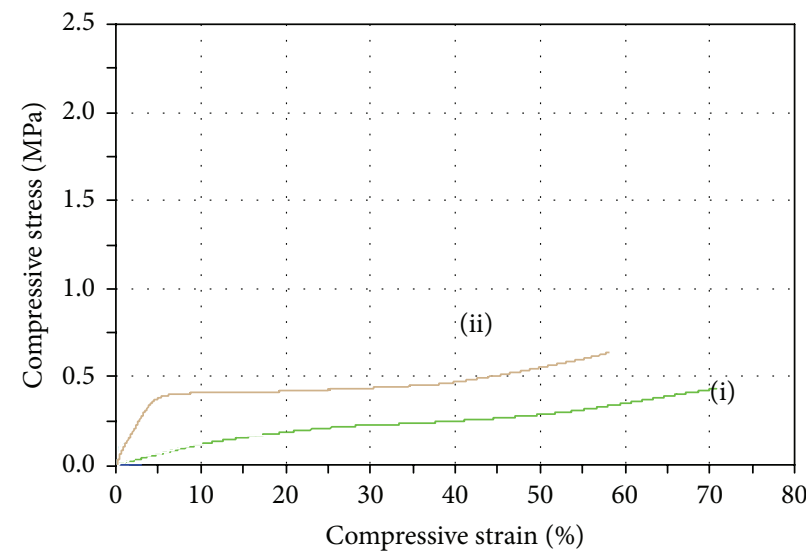

(a)

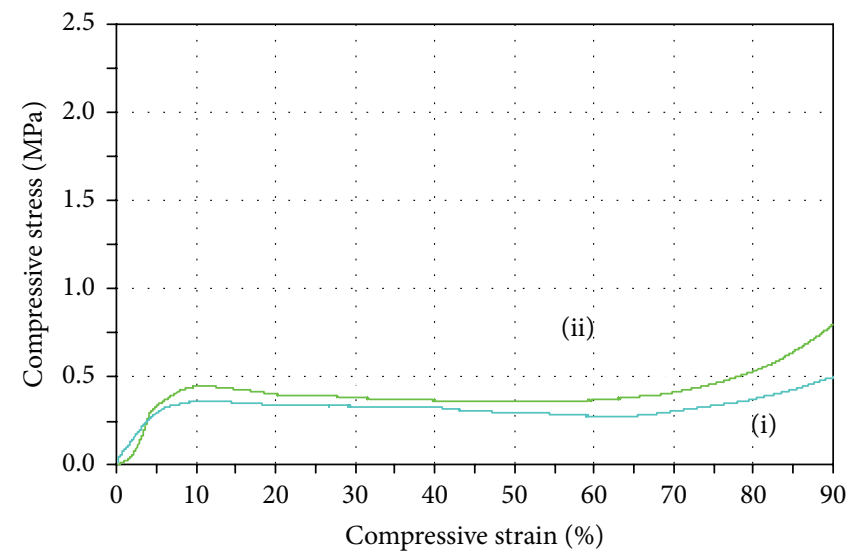

(b)

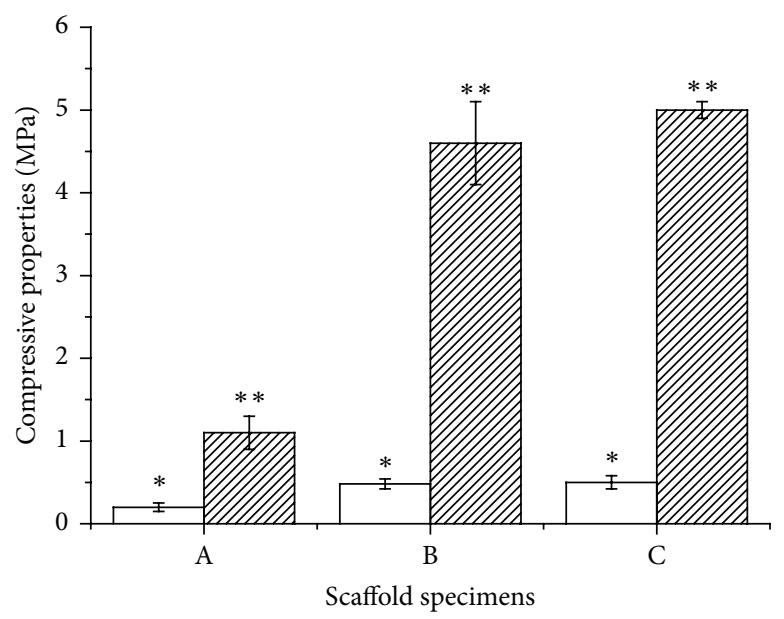

Yield strength

U10 Modulus

(c)

FIGURE 6: (a) Compressive stress-strain curves of (i) CS and (ii) nHA/CS scaffolds; (b) compressive stress-strain curves of (i) nHA/CS and (ii) PEDOT:PSS/nHA/CS scaffolds; (c) compressive yield strength (MPa) and compressive modulus (MPa) of CS (A), nHA/CS (B), and PEDOT:PSS/nHA/CS (C) scaffolds.

TABLE 2: Water contact angles of nonporous thin films produced from CS, nHA/CS, and PEDOT:PSS/nHA/CS at different time points.

Scaffold


$\left.{ }^{* *} p<0.05\right)$. These data demonstrate the positive effects of the addition of HA and PEDOT:PSS in enhancing the mechanical properties of the scaffolds. It was also found that the compressive modulus and yield strength of the $\mathrm{HA} /$ chitosan scaffold were not significantly different from those of HA/chitosan/PEDOT:PSS $(p<0.05)$. From one sample $t$-test, it was found that the moduli of the scaffolds with the same polymer concentration were significantly different $(p<0.05)$. This demonstrates the mechanically anisotropic behavior of the scaffolds fabricated by this technique.

3.3. Swelling and Weight Loss Measurement. The swelling behavior of the scaffolds is presented in Figure 7. After a 24-hour incubation at $37^{\circ} \mathrm{C}$ in $\mathrm{PBS}$, the water uptake of the pure chitosan scaffold was the highest, followed by the nHA/CS and the PEDOT:PSS/nHA/CS scaffolds. With the incorporation of nHA and PEDOT:PSS, the pore sizes and porosity decreased, which influenced the decreasing swelling ratio. The swelling ratio decreased threefold after the addition of nHA and PEDOT:PSS.

PEDOT is an insoluble material in water, which associates with PSS to change its properties. The PSS chains isolate the PEDOT chains and produce a homogeneous conductive core in the grains [32]. The grains of PEDOT:PSS are distributed through the chitosan matrix and the PSS layer interacts most closely with the surrounding PBS. The swelling behavior affects the mechanical properties of the scaffolds. The scaffold can be loosened from its implant site and exert unusual stress on surrounding tissues due to excessive swelling. In this investigation, it was observed that the incorporation of nHA and PEDOT:PSS produced scaffolds with reduced swelling properties compared with the pure CS scaffold. The composite scaffolds had higher compressive mechanical strengths than the CS scaffold.

3.3.1. Weight Loss. The weight loss of the scaffolds was examined in PBS solution. As observed from Figure 7, the CS and nHA/CS scaffolds showed approximately $30 \%$ weight loss after one month, whereas the PEDOT:PSS/nHA/CS scaffold had only $10 \%$ weight loss. With the incorporation of PEDOT:PSS, the stability of the composite scaffold was increased approximately threefold. Based on previous research, the bonding between PEDOT:PSS and the other substrate chains is strong, and particles are seldom produced in composite scaffolds [12].

3.4. Cell Attachment and Cell Morphology. The CS, nHA/CS, and PEDOT:PSS/nHA/CS scaffolds were studied for cell attachment (Figure 8), which is a crucial step in studying the cell behavior. In Figure 8(a), it can be observed that the cell attachment was much higher in the PEDOT:PSS/nHA/CS scaffold than the other scaffolds. The higher cell attachment in the PEDOT:PSS-containing scaffold might be due to the binding properties of the surface of the PEDOT:PSScontaining scaffold and its mechanical compliance, which increased the cell adhesion $[33,34]$. It was reported that the protein-binding properties of the surface can be modulated by the addition of PEDOT [33], which has a direct influence

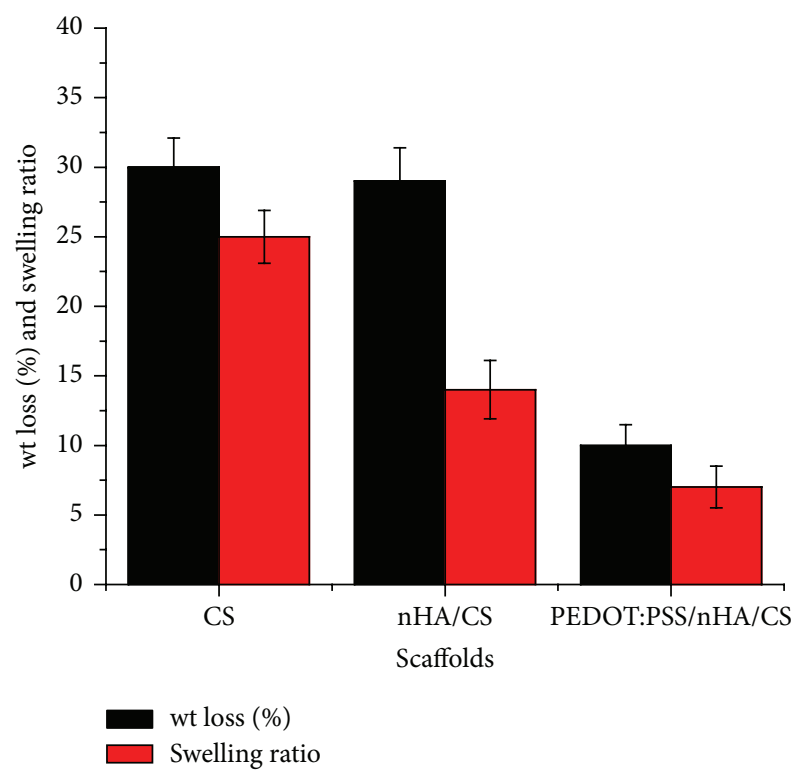

Figure 7: The weight loss and swelling ratio analysis of the CS; nHA/CS; PEDOT:PSS/nHA/CS scaffolds.

on cell adhesion. It was also reported that applying electrical stimuli through conductive polymers can enhance tissue regeneration because of specific interactions between CPs and biological molecules, which serve as conducting biointerfaces [34].

It was revealed from the live/dead assay that, at day 7, the cells grew to the confluence level, and a monolayer of cells was observed on the PEDOT:PSS/nHA/CS scaffold (Figures $8(\mathrm{~b})$ and $8(\mathrm{c})$ ). It was also observed from the live/dead assay that over $98 \%$ of the living cells adhered to the scaffold. The cells were viable as well as metabolically active. The interaction between cells and scaffolds was favorable. The SEM micrographs revealed that the cells had a spindle-like morphology on the surface of the scaffold (Figures $8(\mathrm{~d})$ and $8(\mathrm{e})$ ). On the surface of the scaffold, the cells were anchored by their stretched filopodia. Several nodules were also found on the surfaces of the cells. The cells were well attached on the scaffold surface. Other researchers also observed the positive response of PEDOT:PSS toward cells when using different types of polymers and cells $[12,29]$. The bionanocomposite PEDOT:PSS/nHA/CS scaffold contains bioactive nHA and conductive PEDOT:PSS, which can enhance cell metabolism. The results of the cell viability and morphology of the cells suggested highly active cells, which might be because of the enhanced intracellular electrical signaling. A separate study reported that PEDOT:PSScoated electrospun PHBV/PLA scaffolds exhibited significantly higher cell viability, cell attachment, and proliferation [29]. Another study reported that the hydrophilic and electrical properties are advantageous for cell adhesion [35].

The microstructure of the bionanocomposite scaffold is also propitious for cell growth. The range of pore sizes $(50-350 \mu \mathrm{m})$ and high porosity $(\sim 74 \%)$ offer a considerable 


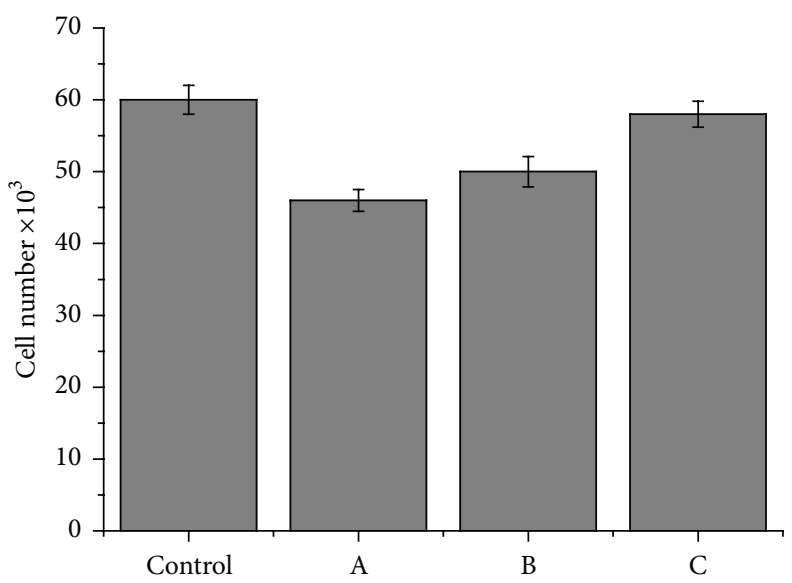

(a)

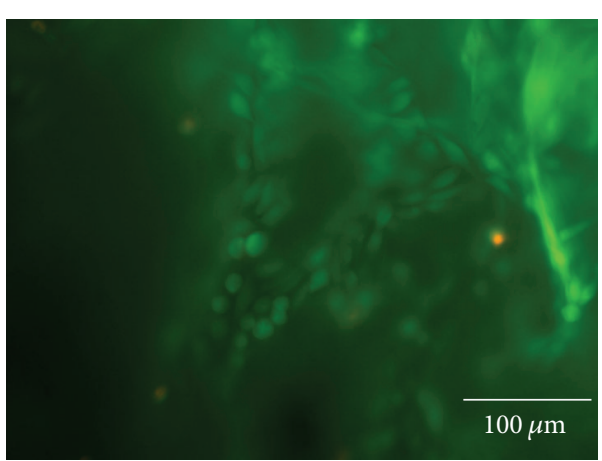

(b)

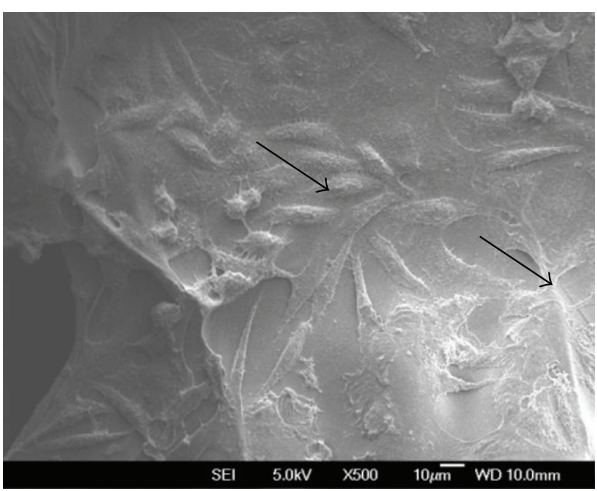

(d)

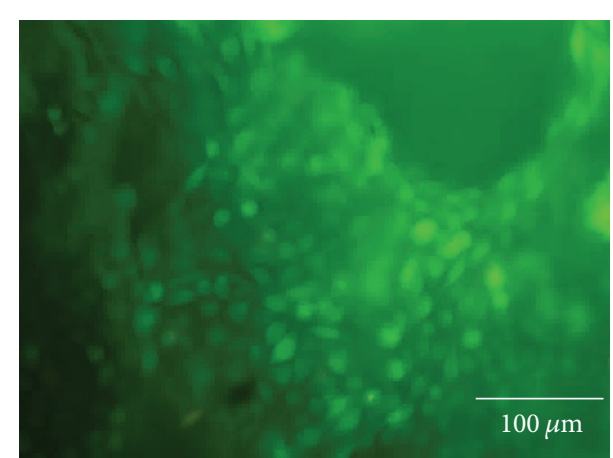

(c)

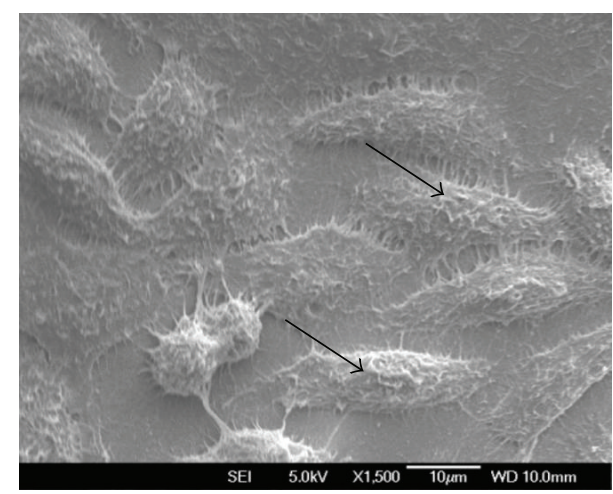

(e)

FIGURE 8: (a) Cells attachment of L929 cells on (A) CS; (B) nHA/CS; and (C) PEDOT:PSS/nHA/CS scaffolds in comparison to control (tissue culture plate) after 24 hours; (b, c) fluorescent microscope image; and (d, e) FESEM micrographs of cells attached to PEDOT:PSS/nHA/CS scaffold.

surface area, which is required for cell growth. The diffusion of metabolic nutrients could be facilitated, and the removal of metabolic wastes could be increased, due to the larger pore size, porosity, and pore interconnectivity [12]. The mechanical properties of the scaffolds potentially offer resistance against breakdown during cell culture in vitro. The PEDOT:PSS residues can be disposed of by the human body. Detailed study of cell attachment and proliferation using osteoblast-type cells is currently underway and will be reported separately.

\section{Conclusions}

Bionanocomposite conductive scaffolds were fabricated using PEDOT:PSS/nHA/CS, and the scaffold properties were evaluated and compared with those of CS and nHA/CS scaffolds. The scaffold's microstructure, conductivity, wettability, and surface roughness were measured. PEDOT:PSS rendered the scaffolds electrically conductive. The PEDOT:PSS/nHA/CS scaffold exhibited slower weight loss and reduced water uptake. Favorable responses during in vitro studies of 
cell viability and cell attachment were achieved using the PEDOT:PSS scaffold. Based on the comparative assessment of the porosity, microstructure, conductivity, and cell responses, PEDOT:PSS/nHA/CS can be regarded as a promising scaffold for tissue engineering applications.

\section{Additional Points}

Highlights. (i) PEDOT:PSS incorporation did not change the microstructure of the scaffold. (ii) PEDOT:PSS imparted conductivity to the composite scaffold. (iii) Swelling ratio and weight loss decreased in the PEDOT:PSS/nHA/CS scaffold. (iv) The PEDOT:PSS-containing nHA/CS scaffold was nontoxic to cells.

\section{Competing Interests}

The authors declare that there is no conflict of interests.

\section{Acknowledgments}

The authors acknowledge MOHE, GUP Tier 1 grants (Vot: 12H24), FRGS (Vot: 4F507, MOHE), Hi COE grant (Vot: 4J191), UTM, and RMC for financial support. Lab facilities of FBME, AMTEC, and UTM centralized lab are also acknowledged.

\section{References}

[1] W. W. Thein-Han and R. D. K. Misra, "Biomimetic chitosannanohydroxyapatite composite scaffolds for bone tissue engineering," Acta Biomaterialia, vol. 5, no. 4, pp. 1182-1197, 2009.

[2] Q. Yao, Y. Yang, X. Pu et al., "Preparation, characterization and osteoblastic activity of chitosan/polycaprolactone/in situ hydroxyapatite scaffolds," Journal of Biomaterials Science, Polymer Edition, vol. 23, no. 14, pp. 1755-1770, 2012.

[3] J. Venkatesan and S.-K. Kim, "Chitosan composites for bone tissue engineering-an overview," Marine Drugs, vol. 8, no. 8, pp. 2252-2266, 2010.

[4] J. Venkatesan, B. Ryu, P. N. Sudha, and S.-K. Kim, "Preparation and characterization of chitosan-carbon nanotube scaffolds for bone tissue engineering," International Journal of Biological Macromolecules, vol. 50, no. 2, pp. 393-402, 2012.

[5] J. M. Oliveira, M. T. Rodrigues, S. S. Silva et al., "Novel hydroxyapatite/chitosan bilayered scaffold for osteochondral tissueengineering applications: scaffold design and its performance when seeded with goat bone marrow stromal cells," Biomaterials, vol. 27, no. 36, pp. 6123-6137, 2006.

[6] Y. Li, T. Liu, J. Zheng, and X. Xu, "Glutaraldehyde-crosslinked chitosan/hydroxyapatite bone repair scaffold and its application as drug carrier for icariin," Journal of Applied Polymer Science, vol. 130, no. 3, pp. 1539-1547, 2013.

[7] E. Fukada and I. Yasuda, "On the piezoelectric effect of bone," Journal of the Physical Society of Japan, vol. 12, no. 10, pp. 1158$1162,1957$.

[8] N. K. Guimard, N. Gomez, and C. E. Schmidt, "Conducting polymers in biomedical engineering," Progress in Polymer Science, vol. 32, no. 8-9, pp. 876-921, 2007.

[9] M. Mozafari, D. Vashaee, L. Tayebi, and M. Mehraien, Electroconductive Nanocomposite Scaffolds: A New Strategy into Tissue
Engineering and Regenerative Medicine, InTech, Rijeka, Croatia, 2012.

[10] M. Li, Y. Guo, Y. Wei, A. G. MacDiarmid, and P. I. Lelkes, "Electrospinning polyaniline-contained gelatin nanofibers for tissue engineering applications," Biomaterials, vol. 27, no. 13, pp. 2705$2715,2006$.

[11] S.-C. Luo, E. Mohamed Ali, N. C. Tansil et al., "Poly(3,4-ethylenedioxythiophene) (PEDOT) nanobiointerfaces: thin, ultrasmooth, and functionalized PEDOT films with in vitro and in vivo biocompatibility," Langmuir, vol. 24, no. 15, pp. 8071-8077, 2008.

[12] A. Shahini, M. Yazdimamaghani, K. J. Walker et al., "3D conductive nanocomposite scaffold for bone tissue engineering," International Journal of Nanomedicine, vol. 9, no. 1, pp. 167-181, 2014.

[13] L. Groenendaal, F. Jonas, D. Freitag, H. Pielartzik, and J. R. Reynolds, "Poly(3,4-ethylenedioxythiophene) and its derivatives: past, present, and future," Advanced Materials, vol. 12, no. 7, pp. 481-494, 2000.

[14] T. Sun, T. H. Khan, and N. Sultana, "Fabrication and in vitro evaluation of nanosized hydroxyapatite/chitosan- based tissue engineering scaffolds," Journal of Nanomaterials, vol. 2014, Article ID 194680, 8 pages, 2014.

[15] N. Sultana, M. Mokhtar, M. I. Hassan, R. M. Jin, F. Roozbahani, and T. H. Khan, "Chitosan-based nanocomposite scaffolds for tissue engineering applications," Materials and Manufacturing Processes, vol. 30, no. 3, pp. 273-278, 2015.

[16] A. Di Martino, M. Sittinger, and M. V. Risbud, "Chitosan: a versatile biopolymer for orthopaedic tissue-engineering," Biomaterials, vol. 26, no. 30, pp. 5983-5990, 2005.

[17] R. Chen, C. Huang, Q. Ke, C. He, H. Wang, and X. Mo, "Preparation and characterization of coaxial electrospun thermoplastic polyurethane/collagen compound nanofibers for tissue engineering applications," Colloids and Surfaces B: Biointerfaces, vol. 79, no. 2, pp. 315-325, 2010.

[18] J.-Y. Je and S.-K. Kim, "Water-soluble chitosan derivatives as a BACE1 inhibitor," Bioorganic \& Medicinal Chemistry, vol. 13, no. 23, pp. 6551-6555, 2005.

[19] M. I. Hassan, T. Sun, and N. Sultana, "Fabrication of nanohydroxyapatite/Poly(caprolactone) composite microfibers using electrospinning technique for tissue engineering applications," Journal of Nanomaterials, vol. 2014, Article ID 209049, 7 pages, 2014.

[20] R. Ma, L. Weng, X. Bao, S. Song, and Y. Zhang, "In vivo biocompatibility and bioactivity of in situ synthesized hydroxyapatite/polyetheretherketone composite materials," Journal of Applied Polymer Science, vol. 127, no. 4, pp. 2581-2587, 2013.

[21] J. Li, Y. Chen, Y. Yin, F. Yao, and K. Yao, "Modulation of nanohydroxyapatite size via formation on chitosan-gelatin network film in situ," Biomaterials, vol. 28, no. 5, pp. 781-790, 2007.

[22] W. Y. Zhou, M. Wang, W. L. Cheung, B. C. Guo, and D. M. Jia, "Synthesis of carbonated hydroxyapatite nanospheres through nanoemulsion," Journal of Materials Science: Materials in Medicine, vol. 19, no. 1, pp. 103-110, 2008.

[23] I. Armentano, M. Dottori, E. Fortunati, S. Mattioli, and J. M. Kenny, "Biodegradable polymer matrix nanocomposites for tissue engineering: a review," Polymer Degradation and Stability, vol. 95, no. 11, pp. 2126-2146, 2010.

[24] I. Yamaguchi, S. Itoh, M. Suzuki, M. Sakane, A. Osaka, and J. Tanaka, "The chitosan prepared from crab tendon I: the characterization and the mechanical properties," Biomaterials, vol. 24, no. 12, pp. 2031-2036, 2003. 
[25] J.-W. Wang, C.-Y. Chen, and Y.-M. Kuo, "Preparation and characterization of chitosan-coated hydroxyapatite nanoparticles as a promising non-viral vector for gene delivery," Journal of Applied Polymer Science, vol. 121, no. 6, pp. 3531-3540, 2011.

[26] K. I. Seo and I. J. Chung, "Reaction analysis of 3,4-ethylenedioxythiophene with potassium persulfate in aqueous solution by using a calorimeter," Polymer, vol. 41, no. 12, pp. 4491-4499, 2000.

[27] E. Min, K. H. Wong, and M. H. Stenzel, "Microwells with patterned proteins by a self-assembly process using honeycombstructured porous films," Advanced Materials, vol. 20, no. 18, pp. 3550-3556, 2008.

[28] D. D. Deligianni, N. D. Katsala, P. G. Koutsoukos, and Y. F. Missirlis, "Effect of surface roughness of hydroxyapatite on human bone marrow cell adhesion, proliferation, differentiation and detachment strength," Biomaterials, vol. 22, no. 1, pp. 87-96, 2001.

[29] H. C. Chang, T. Sun, N. Sultana, M. M. Lim, T. H. Khan, and A. F. Ismail, "Conductive PEDOT:PSS coated polylactide (PLA) and poly(3-hydroxybutyrate-co-3-hydroxyvalerate) (PHBV) electrospun membranes: fabrication and characterization," Materials Science and Engineering: C, vol. 61, pp. 396-410, 2016.

[30] L. J. Gibson and M. F. Ashby, Cellular Solids: Structure and Properties, Cambridge University Press, New York, NY, USA, 2nd edition, 1997.

[31] N. Sultana and M. Wang, "PHBV/PLLA-based composite scaffolds fabricated using an emulsion freezing/freeze-drying technique for bone tissue engineering: surface modification and in vitro biological evaluation," Biofabrication, vol. 4, no. 1, Article ID 015003, 2012.

[32] X. Crispin, S. Marciniak, W. Osikowicz et al., "Conductivity, morphology, interfacial chemistry, and stability of poly $(3,4$ ethylene dioxythiophene)-poly(styrene sulfonate): a photoelectron spectroscopy study," Journal of Polymer Science, Part B: Polymer Physics, vol. 41, no. 21, pp. 2561-2583, 2003.

[33] H. Zhao, B. Zhu, S.-C. Luo et al., "Controlled protein absorption and cell adhesion on polymer-brush-grafted poly $(3,4-$ ethylenedioxythiophene) films," ACS Applied Materials and Interfaces, vol. 5, no. 11, pp. 4536-4543, 2013.

[34] S.-C. Luo, "Conducting polymers as biointerfaces and biomaterials: a perspective for a special issue of polymer reviews," Polymer Reviews, vol. 53, no. 3, pp. 303-310, 2013.

[35] S. R. Benhabbour, H. Sheardown, and A. Adronov, "Cell adhesion and proliferation on hydrophilic dendritically modified surfaces," Biomaterials, vol. 29, no. 31, pp. 4177-4186, 2008. 

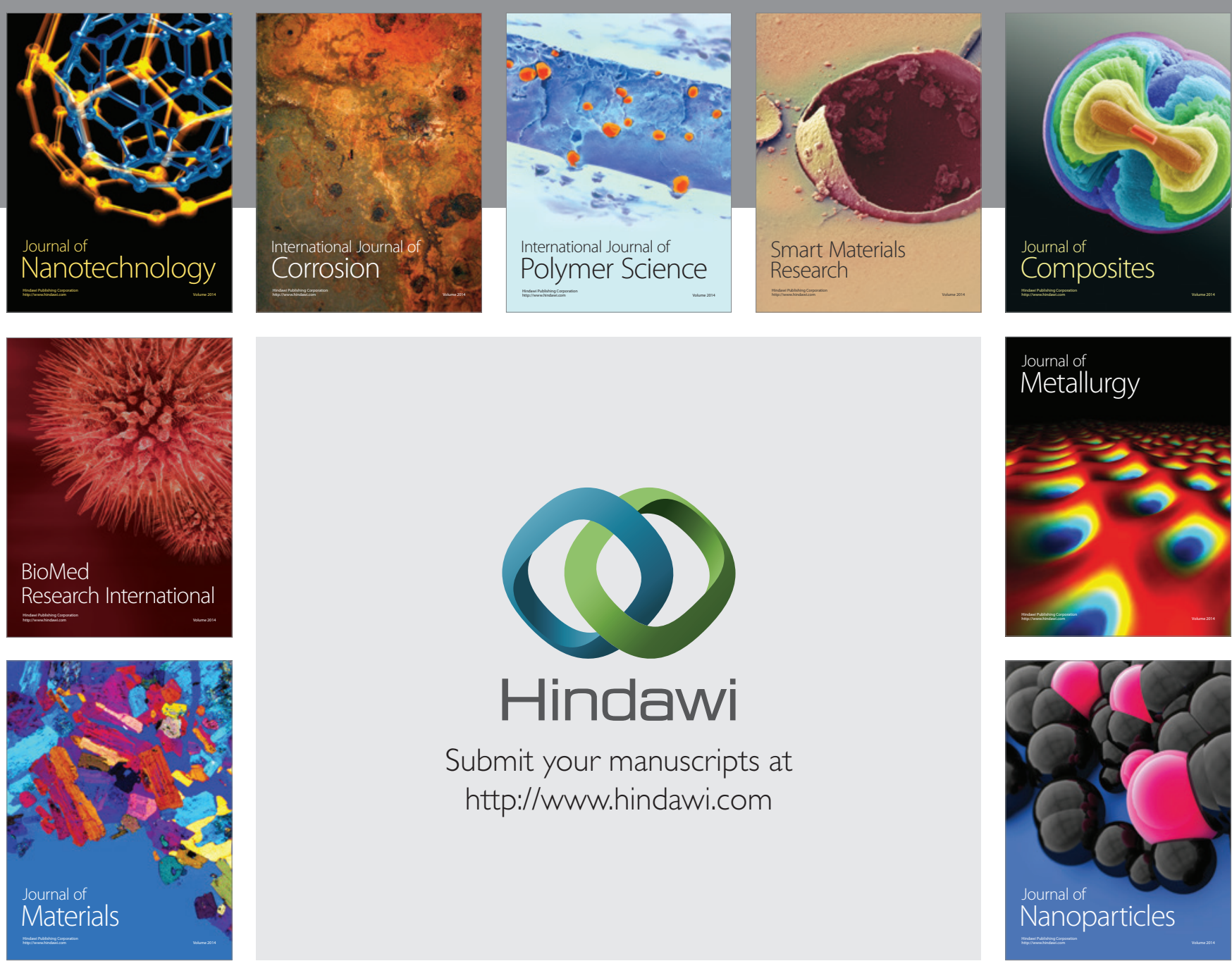

\section{Hindawi}

Submit your manuscripts at

http://www.hindawi.com

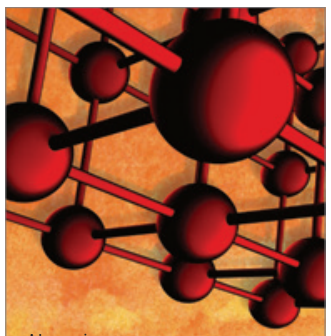

Materials Science and Engineering
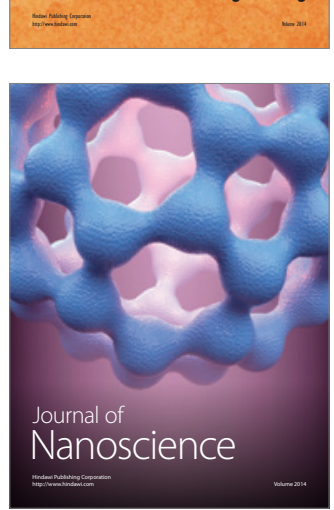
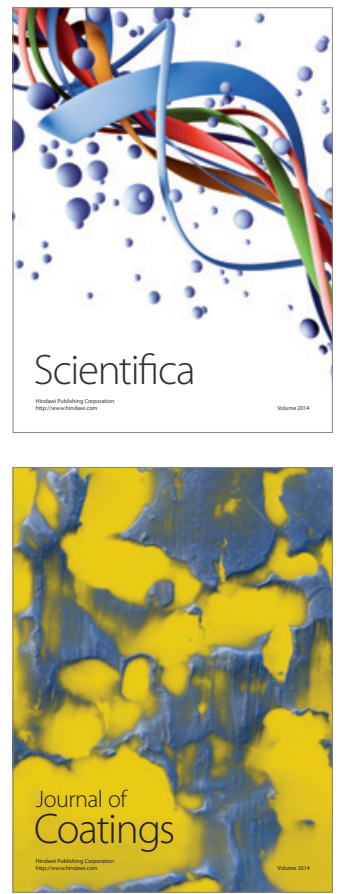
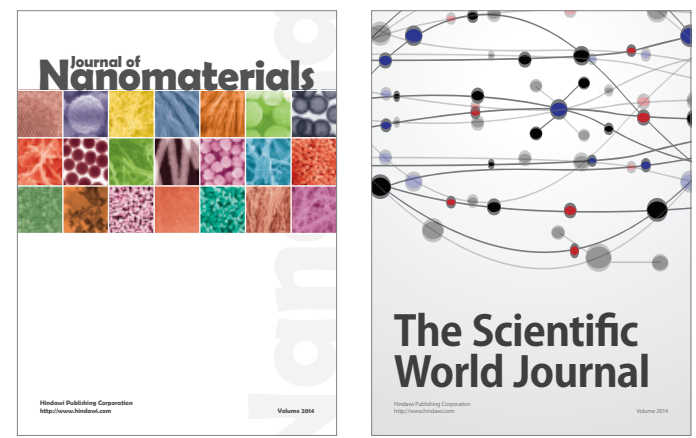

The Scientific World Journal
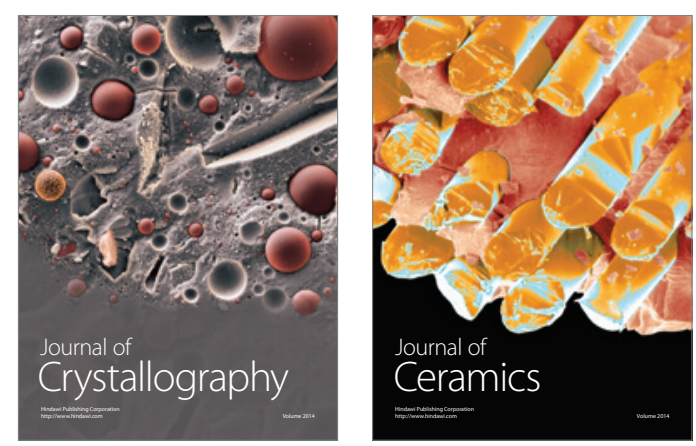
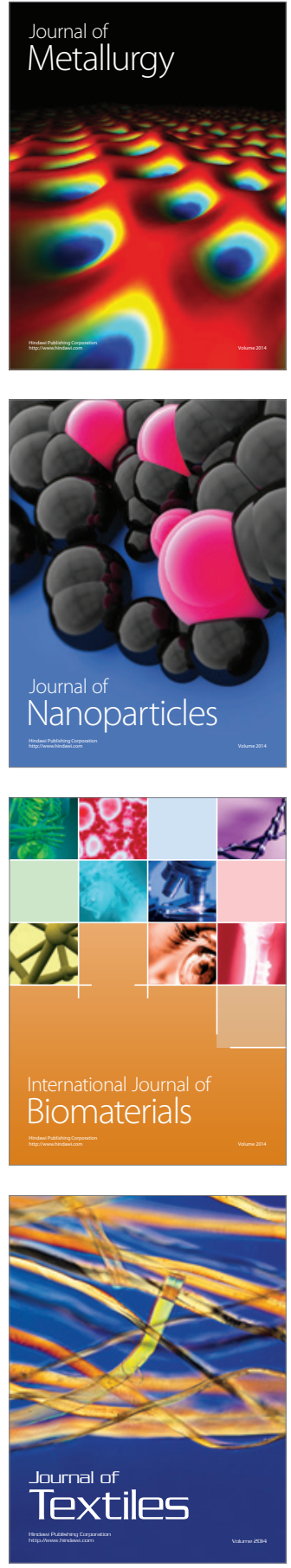